\title{
Stress as the Result of Trauma Situations Experienced by Penitentiary Police (Correction Officers) in the Line of Duty in the Republic of Albania
}

\author{
Colonel Krenar Ahmeti \\ Ph.D. Candidate, Tirana State University; \\ Chief of Special Forces in the Guard of the Republic of Albania \\ Email: krenarahmeti@gmail.com \\ Prof. Dr. Theodhori Karaj \\ Dean, Social Sciences Faculty, Tirana State University \\ Email:dhorikaraj@yahoo.com
}

Doi:10.5901/ajis.2015.v4n2s2p82

\begin{abstract}
This study aims to evidence and explain the connection between stress and trauma situations experienced by penitentiary police or correction officers, viewed as members of those employed to ensure public safety. More specifically, this study has explored what are the trauma situations, their connection to experienced stress, the level of this particular stress and its intensity, etc. That it why the specific OPTI-Fragebogen questionnaire was used as a measuring instrument for public security employees in order to provide a precise expose of the existence of this phenomenon among these kind population, the taxonomic of trauma situation, stress level and the predisposition of this group of population to be affected by the post traumatic stress disorder and psychological aftermath. Ten percent of the general number of efficient correction officers all over the prisons of the Republic of Albania was polled for this questionnaire. This study also sheds light on the need of competent and respective bodies to take preventive measures in order to enable the correction officers and penitentiary police of this institutions to experience as little as possible stress while and after exercising their duties because an employ who enjoys and demonstrates a good state of mental health, will accomplish and fulfill in the best manner his functional tasks. This phenomenon has been widely studied in the most developed countries of the world but it is the first time ever that it is being studied in our country based on rigorously scientific foundations.
\end{abstract}

Keywords: Stress, trauma situation, correction officers, Republic of Albania

\section{Introduction}

To exercise their duties correctional officers (guards) as public safety workers face many different situations that are specific to the profession, the situation giving rise to significant psychological burden, this profession is therefore potentially vulnerable to the psychological burdens as a result of situations related traumatic task. Prison policing is a profession that is as functional task facing different situations as the main ground have prisons and people who were taken prisoners. Being an important profession in matters of public safety, mental health staff is essential, because his own office has a special element of this is direct contact with the community in difficult situations and dangerous.

A number of studies have been conducted in the recent years concerning the tasks troupe and public safety employees. Most of the research comes to conclusion that these institutions face difficult situations on duty.

In addition, stressors in the work of public safety employees may have harmful consequences for physical health and their mental, citing disorders such as post traumatic stress disorder, depression, even suicide aggressive behavior (Blum, 2000; Violanti, 1996).

Very few professions are required to cope with so many dangerous situations and difficult in the performance of functional tasks, as that public safety employees. Therefore being public safety employees is a profession with increasing stressful experiences. This profession that aims to protect public safety through law enforcement has such workers who lose more often traumatic situations in fulfilling his duty related to stress how the performance of duty functional (Rana and Sud, 2008). 


\subsection{Purpose}

It is known that stress can turn into a disorder, if it passes certain levels for an extended period of time and affects the normal functioning of individuals and the performance of public security functionary. The prison police as public safety employees by having a public function, high intensity of contact with inmates who are in the process of rehabilitation, performing functional tasks relatively difficult, should always be in the required ethical and legal. For this reason the measurement of stress parameters empirically optimal solution enables stress management strategies giving precise guidelines for the prevention, management and identification of serious cases and referring them to specialized bodies mental health if deemed necessary.

\subsection{Objectives}

The objectives of this study are:

- Understanding the link between stress and traumatic situations.

- Potential situations that cause stress.

- The intensity of stress experienced.

- The level of this stress and its consequences to public safety employees, in this search is about correctional officers (guards).

\section{Previous theories and on this topic}

The hypothesis of this research study is based on theoretical model of mental disorders associated with: psychopathology and psycho-biology of the disorder, post-traumatic (Horowitz, 1976); Mental Disorders as a result of experiences with violence (Fischer \& Riedessër, 1998); Treatment of psycho-therapeutic context of specific occupations (Flatten, 2001) Strategies of psychological treatment and providing assistance psycho-therapeutic (Bering, 2002): Problems of psychological burden in the professional (Violant \& Aron, 1994); The emergence of symptoms of extreme stress while performing task in which functionary of seriously endangered the life (Robinson, 1997); Psycho-social assistance to public safety officers who have mental health problems as a result of the occupation problematic load (Senkamp \& Martin, 2003).

Physiological damage in combination with mental disorders leads to a greater result of the latter. Risk factors, the feeling of a threat of death, symptoms of intervention and problem-oriented coping, each contributed significantly to the forecast model (Snyder \& Buddeberg, 1996).

Previous research and investigation of the causes of stress have focused mainly on its external causes, specifically in relation to the criteria of duty or organizational impacts or social impacts (Bühler and Land, 2003). Yet the same working conditions might differently affect different individuals, one individual might get psychologically burdened while another might not. Other studies have been conducted to see the correlation between personality predisposition and individual characteristics of public safety employees.

Discussions have focused on analyzing the reasons and motives for becoming a public safety employee. Does it depend on the internal impetus for example "Policeman is born, not made"; or is it for the culture and socialization at work that affects the personality profile of employees, developed by his experiences after he was recruited to the task?

The exposure of workers to stressors numerous and continuous in intensity can harm their performance at work, damage their image and have individual consequences, both physical and mental disorders that might lead to suicide.

Most studies have investigated mainly individual and organizational consequences, the stress, ignoring individual responses and personality as a possible etiology of stress. Other studies instead have investigated the link between both and have identified factors that make an individual vulnerable.

Further other studies refer to the effects of individual characteristics on the experience of the tier of public safety employees, consequently experiencing stress in this population.

Some psychological characteristics are explained by increased risk of Post Traumatic Stress Disorder. Public safety employees with high levels of aggressiveness and low levels of self-efficacy carry more developed symptoms of post traumatic disorders, depression, anxiety and alexythimia (Heinrichs M, Wagner D, W. Schoch, Soravia LM, Hellhammer DH, EHLERT U, 2005).

This may indicate that social support is a factor that protects against various disorders such as depression, mainly by stress, but this means that individuals who have less experience stressful see the actions of other individuals as 
positive and are able to give and receive support from their social network (Regehr C, Hill J, Glancy GD, 2000, Hytten K, Hasle A, 1989, Psarros C, Theleritis CG, Martinaki S, Bergiannaki ID 2008).

Although from a professional perspective, correctional officers run the risk to be exposed to traumatic experiences through their daily work, the prevalence of Post Traumatic Stress Disorder changes when it comes to professions that work in groups, being lower among employees who have the same profile. Individual differences emerge as weakness and flexibility considerably contribute to how intensive and for how long the symptoms last, as a result of traumatic situations related to the way of their experiencing.

A challenge between penitentiary police personnel receive in the study is their unwillingness to seek help for psychological problems. This may be due to a culture and intellectual formation of this population, who know that they have difficulties and weakness or are in a constant pressure to control emotions and a desire to be effective (Penalba V, McGuire H, Leite JR., 2008).

\section{Methodology}

\subsection{Sample}

This study examines in quantitative terms the existence of concerns, mainly emerging in the form of stress, mental disorders prison officers as public safety employees. The study itself combines data from primary and secondary sources using a variety of methods to collect these data.

Through quantitative analysis it was made possible systematic empirical investigation of the data collected from measuring instrument in this case OPTI-Fragebogen questionnaire. Through quantitative analysis it was made possible testing research tasks raised in the study. In this study they conducted a series of analyzes quantitative data collected from 224 questionnaires completed by public security personnel employed by Penitentiary Institutions (prisons). These employees were selected randomly from Penitentiary Institutions geographical reach across the Republic of Albania. This sample represents $10 \%$ of the total number of manpower employed in our country in these institutions.

Table. Geographical scope of Penitentiary Institutions from where the samples were taken.

\begin{tabular}{|l|c|c|c|}
\hline & Frequency & Percentage & Accumulative percentage \\
\hline 302 Penitentiary Institution & 11 & 4.9 & 4.9 \\
313 Penitentiary Institution & 45 & 20.2 & 25.1 \\
325 Penitentiary Institution & 21 & 9.4 & 34.5 \\
Drenova Penitentiary Institution & 36 & 16.1 & 50.7 \\
Fushë-Kruja Penitentiary Institution & 18 & 8.1 & 58.7 \\
Peqin Penitentiary Institution & 45 & 20.2 & 78.9 \\
Shën-Koll Penitentiary Institution & 47 & 21.1 & 100.0 \\
Total & 223 & 100.0 & \\
\hline
\end{tabular}

\subsection{Measuring Instrument}

\subsubsection{Questionnaire}

The questionnaire that was used in this study intents to measure the types of psychological loads in quantitative and qualitative terms, as well as their intensity. The questionnaire used is used to analyze the psychological burdens, worries and stress levels so that public safety employee to help with advice, instructions to avoid these burdens and concerns and to better manage situations that are a source of stress. The main purpose of the construction of this questionnaire is to identify the relatively severe cases to address the specialized institutions which have the capacity for treatment. In publishing their paper (Rohmer \& Rutenfranz, 1975) relied on two basic dimensions: 1 . Dimension of the impact of any traumatic situation, and 2 . Weight of each traumatic load on the psychological situation.

The later questionnaire was reviewed and the questionnaire was designed specifically for public safety employees, which contains a considerable number of psychological burdens, worries and stress that can be experienced and affect mental health as a result of traumatic situations with They face during the performance of duty functional public safety 
employees.

\subsubsection{The dimensions of the questionnaire}

Dimensions of weight loads: Analysis of situations while on duty. Origin of concern (loads of public safety employees, classifications); Time taking reference concern traumatic situation; Subjective perception of the frequency of the phenomenon while on duty (the parameters of formal situation); Subjective evaluation of situations that are considered as a source of psychological concerns of public safety employees while on duty; Loads and personal problems that affect the performance of duty.

\section{Results and Discussions}

This study measures the level of stress and its intensity to public safety employees at Penitentiary Institutions. Obtained in the study sample consisted of 224 persons of which 44 were female and 178 were male. One such change is generally expected since public safety employee is chosen profession by men, and especially when this employee is effective in Penitentiary Institutions, but contingent of prisoners is almost to the same report.

Table 2. Gender of the participants

\begin{tabular}{|lc|c|c|c|c|}
\hline & & Frequency & Percentage & Valuable percentage & Accumulative percentage \\
\hline \multirow{3}{*}{ Gender } & Female & 44 & 19.7 & 19.8 & 19.8 \\
& Male & 178 & 79.8 & 80.2 & 100.0 \\
\multicolumn{2}{|c|}{ Thos who didn't write their gender } & 222 & 99.6 & 100.0 & \\
\multicolumn{2}{|l|}{ Total } & 1 & .4 & & \\
\hline
\end{tabular}

The average age of the sample was 42.3 years, standard deviation 9.1 where the employee with the youngest was 23 years old and older age was 60 years old. This shows a heterogeneous sample in terms of age.

Among other participants in the study were asked about the consequences of stress shown as various symptoms such as anxiety, depression, etc. The questionnaire was coded in such a way that as many points to collect, the more stress symptoms showed public safety employees. The graphic below shows the levels of self-reported symptoms among these employees.

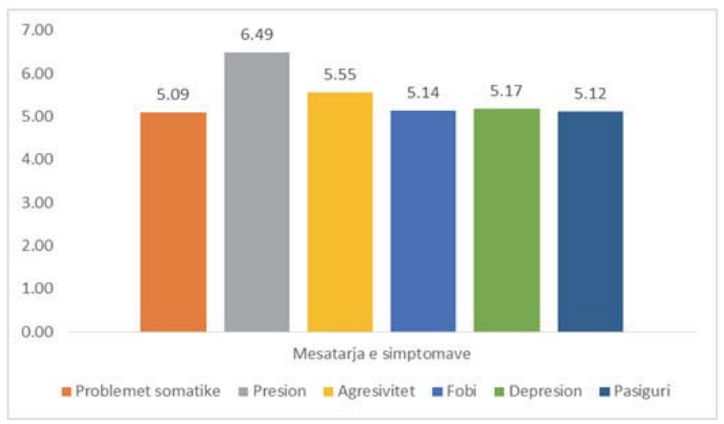

Figure 1. The level of self-reported symptoms of prison employees.

As seen in this graphic, all the symptoms appear more or less at the same level, with the exception of external pressure that has a higher average than others. This may be an indicator that the security staff of Penintetiary Institutions have very high pressure on the type of work they do. 
Another factor that was considered was the intensity of the stress faced by these workers. The chart below shows how the intensity of the work displayed at different levels to employees of Penintetiary Institutions. It notes that almost half of employees claim to have low-intensity stress in their work, but the other half is that the intensity of stress in their work varies from medium to very high. This means that although the tasks of staff in prisons may seem monotonous, without any variation and more routine (especially compared to employees of special teams of riot etc.), again this category has a considerable level of intensity stress facing each day. This intensity has its consequences, which will be discussed below.

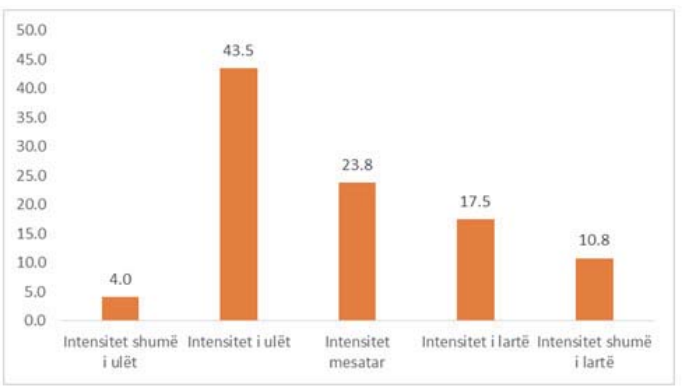

Graphic 2. Stress intensity among the employees of Penintetiary Institutions (Correction Facilities)

The level of intensity of experiencing the stress and symptoms experienced were koreluan and found that the Pearson coefficient was $r=0,402, p<0,01$. This means that by increasing the intensity of stress increase the physical and psychological symptoms of the prison. Based on this correlation was conducted regression analysis which showed that if we have information about the intensity of the stress experienced by employees of prisons, then we can predict the level reported symptoms. The following tables are presented detailed data regression.

Table 3. The regression table on the physical and psychological symptoms based on stress' intensity

Model summary

\begin{tabular}{|c|c|c|c|c|}
\hline Model & $\mathrm{R}$ & Square R & Reviewed square R & Standard error \\
\hline 1 & $.402^{\mathrm{a}}$ & .162 & .158 & 12.4932 \\
\hline
\end{tabular}

a. Predictor: Constant value, physical and psychological symptoms

Coefficients

\begin{tabular}{|l|c|c|c|c|c|}
\hline \multirow{2}{*}{ Model } & \multicolumn{2}{|c|}{ Non standard coefficient } & Standard coefficients & \multirow{2}{*}{$\mathrm{t}$} & Sic. \\
\cline { 2 - 4 } & $\mathrm{B}$ & Std. Error & Beta & & \\
\hline Constant value & 9.729 & 1.666 & & 5.840 & .000 \\
Intensity & 2.504 & .384 & .402 & 6.520 & .000 \\
\hline
\end{tabular}

a. Depended variable: somatic and psychological symptoms

Another analysis was done to see if there were differences between male employees and female prison in connection with the intensity of experienced stress and symptoms shown. For this analysis was conducted independent $t$ test. As can be seen in the table below, men report experiencing more symptoms, although the level of intensity of stress is the same. This may be because women may have better mechanisms of protection to the intensity of their stress .

Table 4. Changes of stress levels and of reported symptoms, according to gender.

\begin{tabular}{|c|c|c|c|c|c|c|c|}
\hline & Gender & $\mathrm{N}$ & Average & Standard deviation & $\mathrm{t}$ & Grades of freedom & $p$ \\
\hline \multirow{4}{*}{ Symptoms } & Female & 44 & 13.545 & 7.8071 & -4.35 & 125 & 0.002 \\
\hline & Male & 178 & 20.492 & 14.3897 & & & \\
\hline & Female & 44 & 3.6818 & 1.97393 & 0.22 & 220 & 0.82 \\
\hline & Male & 178 & 3.7640 & 2.24249 & & & \\
\hline
\end{tabular}


Public safety employees in Albania's Penitentiary Institutions face every day with high stress levels and strong intensity. This kind of situation makes that these employees have different symptoms such as anxiety, depression, etc., and consequently their performance may differ. In this study there was a measure of the performance of staff in prisons, but it is logical to think that an employee with high intensity stress and various symptoms due to this stress will not have the best performance possible.

\section{Discussion}

In this scientific paper discussed basic research and scientific definitions of stress, and illustrated the basic assumptions of common concepts on stress. Also there were taken into consideration the explanatory models of the formation of psychology of stress and factors relating to the performance of functional duties of public safety employees, which affect the experience of stress and its possible effects.

The change of the political system after 1990 brought forth such requests for public security institutions in the context of a pluralistic democratic social system, to which employees were not trained.

Main exigencies were changing the status from military to civilian, changing the concept of work, structure and methodology associated with changes in legislation. This flow of requirements found the Penitentiary Institutions employees unprepared efficiently, both the present at that time staff and also the new staff which would have recruited. Under that climate of social and institutional pressure exercised on the population to carry out its duties with efficiency, substantial growth in the number of traumatic situations to which they were exposed to public safety employees brought an increase in the possibility to be affected by disturbances such as disorders like anxiety disorders, post-traumatic stress and other deviations to serious consequences for mental health.

Fears that the responsibility for the incident lies with the worker safety, redundant administrative tasks, lack of support from the institution, the suppress of emotions and emotional conflict are some of the factors which says the conflict between the demands of duty and available resources. Coping skills and solving situations styles create a barrier which helps in managing problems associated with the performance of duty, but the sources of stress dealing with risk and uncertainty, low wages, and the hostility of the prisoners etc. They remain there and continue to maintain this population as public safety employees under stress on normal values, compared with the community where they live.

Penitentiary Institutions officers who in many cases are the first to face the specific situation of these institutions, especially in the management of situations with inmates, prison revolts, etc., in this way involved in incidents and situations in the fulfillment of their functional duties, thus is a profession that is considered very dangerous and stressful. This population can be exposed to stressors directly and indirectly, as risking his life when they came and operating in incident management to meet the functional task and when they witness such suffering to others attempted suicide of detainees. Even today we do not have the proper structure attached within public security institutions and in particular to prisons, to be responsible for their mental health. This care starts with recruiting, psychological preparation for the task that they will perform and prevention and treatment of disorders that occur during and after the duty to give necessary assistance to this public safety employee.

\section{Conclusions}

Penitentiary Institutions officers as members of public safety employees are not immune from experiencing a higher stress level than the average population and participation in traumatic events during the performance of functional tasks, it can make them more likely to suffer the various disorders mainly ÇSTP. Taxonomy provided in the questionnaire once again confirmed the accuracy of the statement that the employee questionnaire and security are not home, so no matter who does the community (the state) belongs to and the figures show almost the same level.

Further, this population untrained in particular higher risk of experiencing psychological distress than trained personnel. The first selection of the manpower, their preparation up to the optimum level professional to potentially stressful situations, can have good benefits. Some personality traits, such as a history of psychiatric morbidity and poor social support might increase the risk for various mental disorders mainly ÇSTP. Stress level can decrease if some preventive factors are in place such as the environment in the work place and support from staff and colleagues.

\section{Recommendations}

There are three main prevention strategies after a traumatic event in the development of mental disorders: 
1. pre-employment selection,

2. training in stress management

3. early intervention.

Employees who have been trained previously and with care seem to handle it better compared to non-experts. Preparing to manage stress is important for staff that has a high risk to be affected by traumatic stress disorder. Here, employees are made known potential experiences during traumatic situations and instructed to manage stress and other psychological concerns them. So the genuine program of stress management, experience in handling stress, dealing with sports and fitness activities can greater preserve the welfare and wellbeing of staff.

Early intervention includes a number of treatments and interventions such as psychiatric treatment, mobilization of institution's own resources. In this regard part of this mobilization could be the support of the colleagues, trainings designed to manage traumatic exposure in the acute phase after a traumatic event, the development of a management plan in the workplace. Another intervention recommended is cognitive behavioral therapy especially for those who develop symptoms within 3 months.

\section{References}

Bering, R.; Fischer, G.; Johansen, F.F.: Neurovulnerabilität der Hippokampus-formation bei der posttraumatischen Belastungsstörung: Forschungsstand und Forschungshypothesen. Psychotraumatologie.

[Online: www.thieme.de/psychotrauma] 2 (2002).

Bering, R.; Horn, A.; Fischer, G.: Psychopharmakotherapie der posttraumatischen Belastungsstörung. Psychotraumatologie [Online: www.thieme.de/psychotrauma] 1 (2002)

Beaton RD, Murphy SA. Sources of occupational stress among firefighter/EMTs and firefighter/paramedics and correlations with jobrelated outcomes. Prehosp Disaster Med 1993; 8:140-150.

Corneil W, Beaton R, Murphy S, Johnson C, Pike K. Exposure to traumatic incidents and prevalence of posttraumatic stress symptomatology in urban firefighters in two countries. J Occup Health Psychol 1999; 4:131-141.

Flatten, G.; Reddemann, L.; Wöller, W.; Hofman, A.: Therapie der Posttraumatischen Belastungsstörung. In: Flatten, G.; Gast, U.; Hofmann, A.; Liebermann, P.; Reddemann, L.; Siol, T.; Wöller, W.; Petzold, E. R. (Hrsg.): Posttraumatische Belastungsstörung. Leitlinie und Quellentext. Stuttgart: Schattauer 2001.

Heinrichs M, Wagner D, Schoch W, Soravia LM, Hellhammer DH, Ehlert U. Predicting posttraumatic stress symptoms from pretraumatic risk factors: a 2-year prospective follow-up study in firefighters. Am J Psychiatry 2005; 162:2276-2286.

Horowitz, M.J.: Stress response syndromes. New York: Aronson 1976

Horowitz, M.J.; Wilner, N.; Alvarez, W.: Impact of Event Scale: a measure of subjective stress. Psychosomatic Medicine 41 (1979), 209218

Lazarus, R.S.; Averill, J.R.: Emotion and cognition: With special references to anxiety. In: Spielberger, C.D. (Hrsg.): Anxiety: Current trends in theory and research. New York: Academic press 1972

McFarlane AC. The aetiology of post-traumatic morbidity: predisposing, precipitating and perpetuating factors. Br J Psychiatry 1989; 154:221-228.

Berger W, Coutinho ES, Figueira I et al. Rescuers at risk: a systematic review and meta-regression analysis of the worldwide current prevalence and correlates of Post Trauma Disorder in rescue workers. Soc Psychiatry Psychiatr Epidemiol 2012; 47:1001-1011.

MacDonald HA, Colotla V, Flamer S, Karlinsky H. Posttraumatic stress disorder (Post Traumatic Stress Disorder) in the workplace: a descriptive study of workers experiencing Post Traumatic Stress Disorder resulting from work injury. J Occup Rehabil 2003; 13:63-77.

Penalba V, McGuire H, Leite JR. Psychosocial interventions for prevention of psychological disorders in law enforcement officers. Cochrane Database of Syst Rev 2008: CD005601. doi:10.1002/14651858.CD005601.pub2.

Podsakoff PM, MacKenzie SB, Lee JY, Podsakoff NP. Common method biases in behavioral research: a critical review of the literature and recommended remedies. J Appl Psychol 2003; 88:879-903.

Robinson, H.M.; Sigman, M.R.; Wilson, J.P.: Duty-related stressors and PTSD symptoms in surburban police officers. Psychological Reports 81 (1997), 835-845.

Violanti, J.; Aron, F.: Ranking Police Stressors. Psychological Reports 75 (1994), 824-826.

Weisæth L. Dalgard OS. Mental Health: Risk Factors and Prevention. Oslo: Gyldendal Akademisk, 2000; 205-236. Norwegian. 